\title{
La Propiedad Industrial En La Sociedad Del Conocimiento. Tendencias Actuales
}

\author{
Eduardo Vinicio Mejía Chávez, Mgr. \\ Facultad de Ciencias Políticas y Administrativas \\ Universidad Nacional de Chimborazo, Ecuador
}

Doi: 10.19044/esj.2017.v13n29p216 URL:http://dx.doi.org/10.19044/esj.2017.v13n29p216

\begin{abstract}
Today humanity is immersed in the knowledge society, so that intangibles have acquired great value throughout the world. This has contributed to globalization and the emergence of economies founded on knowledge and technological development, which have led to the emergence of new intellectual products that generate industrial property rights. The ownership of these industrial property rights has determined the development and economic advancement of countries, but also an absolutely heterogeneous growth between them, as a differentiating element is contemplated, the science and technology they possess. It is therefore in permanent debate whether industrial property is a mechanism of technological development, based on economic recognition of the institutions and their inventors for the science and technology they generate, or whether it is a form of privatization of knowledge and a new Technological colonialism.
\end{abstract}

Keywords: Industrial property, knowledge society, technological development

\section{Resúmen}

Actualmente la humanidad se encuentra inmersa en la sociedad del conocimiento, por lo que los intangibles han adquirido gran valor en todo el mundo. Para ello ha contribuido la globalización y la aparición de economías fundadas en el conocimiento y el desarrollo tecnológico, que han motivado la aparición de nuevos productos intelectuales que generan derechos de propiedad industrial. La titularidad de estos derechos de propiedad industrial ha determinado el desarrollo y adelanto económico de los países, pero también un crecimiento absolutamente heterogéneo entre ellos, como elemento diferenciador se contempla, la ciencia y la tecnología que poseen. Por tanto, está en debate permanente si la propiedad industrial es un 
mecanismo de desarrollo tecnológico, a partir del reconocimiento económico a las instituciones y a sus inventores por la ciencia y tecnología que generan, o si es una forma de privatización del conocimiento y de un nuevo colonialismo tecnológico.

Palabras-clave: Propiedad industrial, sociedad del conocimiento, desarrollo tecnológico

\section{Introducción}

En las últimas décadas el conocimiento ha desempeñado un papel relevante y trascendental en el desarrollo de las sociedades y los países, con la aparición de economías basadas en el conocimiento y una constante revolución tecnológica, son los nuevos escenarios que configuran modelos y formas de vida; al respecto, existen posturas en la que señalan que el conocimiento es la moneda de la nueva economía (Altés, 2015).

En este ámbito, constantemente se incrementa la importancia de la generación de conocimiento en base a lo cual se desarrollan los países y en muchos casos también se incrementan las desigualdades, entre quienes tienen acceso a la generación de conocimiento y aquellos que tienen limitaciones en este tema; tal es su influencia, que en la actualidad se presenta un marcado crecimiento absolutamente heterogéneo de los países en función del conocimiento, la ciencia y la tecnología que poseen. Del mismo modo que el crecimiento tiene que ver cada vez más con el uso programado de la ciencia y la tecnología, el subdesarrollo y la pobreza tiene que ver con el conocimiento. Hace menos de diez años esto aún no parecía claro. Los países pobres pagaban asesores que cobraban costos muy altos, pero no organizaban la simbiosis entre la educación, la economía, la ciencia, la tecnología y la sociedad. Corea tuvo la lucidez para hacerlo y tuvo éxito. Tras ella siguieron Singapur, Tailandia, Malasia. Fueron saliendo de la pobreza, mientras países ricos, como Argentina o Brasil, se fueron hundiendo cada vez más en ella por una mala articulación de sus recursos humanos, económicos, educativos, científicos y tecnológicos (Pérez Lindo, 1998).

Los países poseedores del conocimiento, científica y tecnológicamente desarrollados, sin duda, lo aprovechan mediante la explotación de la titularidad de los derechos de propiedad industrial, excluyendo del acceso al conocimiento a segmentos y colectivos que no poseen los recursos financieros para beneficiarse de ellos mediante licenciamiento o adquiriendo su titularidad. En consecuencia, el problema científico se define y plantea en los siguientes términos: Los derechos que genera la propiedad industrial, son una forma de privatización del conocimiento que contribuyen al desarrollo heterogéneo de las naciones. 
De acuerdo con el problema planteado, los objetivos que plantea la investigación son: Analizar las particularidades de propiedad industrial en la sociedad del conocimiento y las tendencias actuales. Así también, analizar el papel que juega la propiedad industrial frente al desarrollo de las naciones y a la apropiación particular de la ciencia y tecnología.

La investigación se propone dar respuesta a las siguientes interrogantes científicas:

a) ¿Los derechos de propiedad intelectual han contribuido al crecimiento heterogéneo de las naciones?

b) ¿Son los derechos de propiedad intelectual una forma de privatización del conocimiento?

En concordancia con los objetivos y las preguntas científicas formuladas en la investigación, los resultados de la investigación se centran en: Analizar las características particulares de la propiedad industrial y el papel que desempeña en la sociedad actual. Por otra parte, determinar si la propiedad industrial es una forma de privatizar el conocimiento y su influencia en el desarrollo de los países.

La relevancia científica de la presente investigación, se expresa en el establecimiento de parámetros que permitan catalogar si la propiedad industrial es una forma de privatizar el conocimiento, que coadyuva al desarrollo heterogéneo de las naciones o si es un mecanismo para la debida protección a los creadores en el orden moral y patrimonial, que permite la transferencia de los resultados investigativos al sector social y empresarial, estimulando el desarrollo investigativo en correspondencia con las necesidades de la economía y el desarrollo. Así, la investigación se constituye en un estudio pionero en Ecuador, y que tiene su utilidad porque es referente para las consultas de estudiantes e investigadores.

Así también, conviene destacar otros aspectos relacionados al aporte de la investigación en el ámbito teórico, ésta definitivamente se constituye una contribución a la ciencia mediante el establecimiento del papel que desempeña la propiedad industrial en la sociedad del conocimiento y en el desarrollo de las naciones; a su vez, en el ámbito social, se establece si los derechos de propiedad industrial son una forma de privatización o un mecanismo idóneo de fomentar la actividad innovadora e investigativa.

\section{Metodología}

Los aspectos metodológicos que contempla la investigación, adopta el Método Histórico-Lógico para evaluar el proceso evolutivo del conocimiento y el comportamiento histórico de los derechos de propiedad industrial, a fin de explicar su estado actual y su importancia; por lo tanto corresponde a una investigación descriptiva analítica de la propiedad industrial que permite valorar las regulaciones de la temática de estudio; así 
también acompala el Método Jurídico Doctrinal que permite analizar las posiciones jurídicas sobre el fenómeno de estudio y las conclusiones respectivas; finalmente el análisis de la literatura y documentos especializados permiten fundamentar con argumentos y experiencias válidas que alimentan los planteamientos y robustecen el tema de estudio.

En este contexto, se presentan algunas cuestiones relacionadas a la teoría, ya que el conocimiento no siempre ha desempeñado un papel trascendental en el desarrollo de la humanidad, si bien históricamente se origina junto con la humanidad, fue superando diferentes etapas de cambios, transformaciones y desarrollo a lo largo de la historia; en este ámbito, conviene resaltar el tránsito de una economía inicial basada en el trabajo manual y la tracción animal, que a mediados del siglo XVIII se transformó en una economía en la que imperó el uso de la máquina para la fabricación industrial, siendo la producción de bienes la base para la generación de riqueza, la misma que posteriormente derivó en una economía basada en el conocimiento, donde aparece la generación de servicios juntamente con la producción de bienes; a partir de entonces, el conocimiento ha ocupado siempre el lugar central en el crecimiento y desarrollo económico y en la mejora del bienestar y calidad de vida de la población, tanto que la capacidad de inventar, innovar, crear nuevos conocimientos e ideas que se materializan en productos, procedimientos y organizaciones, ha alimentado históricamente al desarrollo (David y Foray, 2002).

\section{Desarrollo}

Indudablemente, la sociedad y las instituciones experimentan a diario que pertenecen y viven en una sociedad del conocimiento, o knowledge society en el mundo anglosajón, en base a lo cual los intangibles han adquirido gran valor en todo el mundo, a la vez que la innovación se ha vuelto una prioridad en centros de investigación, universidades y en general en las políticas que implementan los gobiernos para enfrentar un entorno altamente cambiante. El conocimiento y la innovación tecnológica se convierten recursos estratégicos básicos para alcanzar niveles de competitividad y generar ventajas comparativas en el mercado, donde una herramienta fundamental que diferencia y coadyuva en ese propósito, es el desarrollo de nuevos productos y procesos (Alvarez, 1969; López-Cozar y Cuello, 2008; Martínez de Aguirre, 2016).

Según la UNESCO (2005), la noción de "sociedad del conocimiento" fue utilizada por primera vez en 1969 por un universitario Peter Drucker y en el decenio de 1990 fue profundizada en una serie de estudios detallados publicados por investigadores como Robin Mansell o Nico Stehr

En Latinoamérica fue Pablo Belly, quien empezó acuñando el término sociedad del *conocimiento (Belly, 2014), destacando 
acertadamente la importancia que implicaba para los países subdesarrollados "dejar de invertir en el músculo del brazo para invertir más en desarrollar el músculo de la cabeza”. Efectivamente, Belly, a partir de 1994, resaltaba que las naciones que pretendan desarrollarse van a tener que dejar de hacer circular el dinero y sustituir, dicha circulación, por la del conocimiento, porque es éste el que genera recursos económicos en las sociedades postindustriales que les ha correspondido ser parte en un mundo globalizado, dejando atrás a la sociedad preindustrial caracterizada por una economía basada en la agricultura tradicional y la manufactura, que finalmente fue la sociedad industrial que superó las herramientas para dar paso a las máquinas y la tecnología 58 .

El sostenimiento de la sociedad del conocimiento se fundamenta en la actividad investigativa universitaria y de centros privados y públicos, que incorporan conocimientos y tecnologías que se traducen en el establecimiento de productos innovadores, nuevos servicios y otras formas de conocimiento.

Existen claras muestras de la importancia del conocimiento en el desarrollo de las sociedades y sus economías, entre las que se destacan:

a) El incremento notorio y permanente de recursos humanos $y$ económicos en I+D que los gobiernos, las universidades y la industria han destinado.

b) La aparición de actividades económicas basadas en la generación de nuevos conocimientos y el incremento sustancial de algunas existentes, que han permitido dinamizar las economías de las naciones.

c) La transformación de las universidades, especialmente entre los países con menor desarrollo, que pretenden dejar de ser únicamente formadoras de profesionales a ser generadoras de conocimiento.

d) El surgimiento de una amplia clase de "trabajadores de conocimiento (Valhondo, 2010) con alta formación científica y académica, idóneos en el manejo de tecnologías; así como de una nueva clase de consumidores y ciudadanos adaptados a esta nueva realidad, que hace que la educación juegue un rol preponderante.

e) La aparición de una sociedad que basa su desarrollo en la investigación científica y en la educación, que permite la transformación de materiales en bienes y servicios con un alto componente de valor agregado.

58 Históricamente hemos asistido a la sustitución paulatina del trabajo manual por la utilización de máquinas. Este incremento en el uso progresivo de las tecnologías tuvo su eclosión con la invención de la máquina de vapor, la cual nos adentró de lleno en la denominada sociedad industrial. Con posterioridad, el poder de las máquinas se está sustituyendo por el poder del conocimiento, lo que nos conduce a la sociedad actual. (García, 2010) 
f) La simplificación de actividades a través del internet, que evita la movilidad de las personas, privilegiando el movimiento del conocimiento.

g) La aparición constante de nuevos materiales, instrumentos, equipos, procedimientos y en general de nuevas tecnologías.

h) El crecimiento económico, social y político heterogéneo de los países, dado por la aplicación en su provecho de las ventajas que poseen las naciones que generan conocimiento y las exclusiones que su ausencia provoca; lo cual ha determinado la constitución de un injusto sector político hegemónico mundial, que se ha encargado de acentuar la brecha de desarrollo económico y bienestar social, respecto de los países que no generan o poseen conocimiento.

Así, el conocimiento se constituye en un factor decisivo en el ámbito de las ciencias económicas, bienestar social, cultural y político de la humanidad; siendo, además, el principal insumo del proceso económico y productivo (Nuñez, 2012), como señala el índice mundial de innovación 2016, emitido por la Organización Mundial de Propiedad Intelectual (OMPI), en el que se observa que los países con mayor innovación en el orbe, también son países que poseen un crecimiento económico importante y se sitúan en el contexto de los países desarrollados, con altos niveles de bienestar y calidad de vida de su población, estos indicadores lo encabezan países con los mejores resultados en innovación: Suiza, Suecia, Reino Unido, Estados Unidos, Finlandia, Singapur; en cambio, los países de Latinoamérica y el Caribe, lideran Chile, Costa Rica, que aparecen en el puesto 43 y 44, mientras que Brasil se ubica en el puesto 69 y Ecuador en el 100, entre 128 países que conforma el informe de 2016.

El desarrollo e importancia de la innovación se refleja en las solicitudes de patentes presentado en todo el mundo, que alcanza en torno a 2,9 millones de solicitudes, lo que representa un 7,8\% de incremento respecto al 2014 y el sexto año consecutivo de aumento en la demanda de protección por patente; mientras que las solicitudes de registro de marcas aumentaron en un 15,3\%, situándose en cerca de 6 millones en 2015, en tanto que las solicitudes de registro de diseños industriales crecieron en un $2,3 \%$, cifrándose en 872.800 , conforme se indica en el informe anual de la OMPI sobre Indicadores mundiales de propiedad intelectual.

La OMPI señala con mayor énfasis que la propiedad intelectual es uno de los activos más valorados, y a menudo el más importante en las transacciones comerciales (IDRIS, 2013), en virtud a lo cual se han suscrito a lo largo de la historia importantes instrumentos jurídicos que la regulan, destacándose el Acuerdo de la Organización Mundial de Comercio OMC, sobre los aspectos de los Derechos de Propiedad Intelectual relacionados con el Comercio, en contrapartida de la propiedad industrial que abarca patentes, marcas, indicaciones geográficas, indicaciones de origen, dibujos y modelos 
industriales, esquemas de trazados, información no divulgada y secretos comerciales, homogenizando patrones, estableciendo, procedimientos $\mathrm{y}$ recursos internos encaminados a la observancia de los derechos de propiedad intelectual, a más de normas mínimas de protección que ha de prever cada miembro, procedimientos y recursos internos encaminados a la observancia de los derechos de propiedad intelectual, ratificando la imperatividad de cumplimiento de normas fijadas en acuerdos anteriores y recogiendo principios fundamentales y algunas normas generales encaminadas a evitar dificultades de procedimiento para adquirir o mantener los derechos de propiedad intelectual.

Así también el éxito para muchas organizaciones depende de su capacidad para obtener recursos financieros, que en gran medida giran en base a su capital tecnológico, autores como Bueno (2011), señala que es el conjunto de intangibles vinculados con el desarrollo de las actividades y funciones del sistema técnico de una organización, responsables de la obtención de bienes y servicios, de la implementación de procesos y producción eficiente y del avance en la base necesaria para el desarrollo de futuras innovaciones en productos y procesos.

Respecto del sector empresarial, se puede afirmar que debido a los cambios que existen producto a la globalización, las organizaciones deben tener cada vez más conciencia respecto al conocimiento y su implementación práctica; se afirma que el intercambio de conocimientos es fundamental para el éxito y la supervivencia de las empresas (Casado, 1991; Rogel, 2004), y es esencial para facilitar la toma de decisiones en inversiones, siendo determinante, también, en el desarrollo de la economía mundial, que gira, en gran medida, en torno al aumento de generación de conocimiento (Caryannis, 2016), como se puede apreciar en el número de aplicaciones de patentes presentadas en la OMPI, por ejemplo en el periodo 2004 a 2014 se incrementó de 1.574 .300 a 2.680.900 solicitudes en una década, mientras que en modelos de utilidad el incremento fue de 215.200 a 948.900 solicitudes presentadas, lo cual evidencia un crecimiento destacable.

En este contexto, la generación constante de innovaciones marca el desarrollo y estructura de la sociedad actual; es así, que la producción de conocimiento determina el emerger y el establecimiento de creaciones de productos industriales, conocimientos técnicos o know-how y signos distintivos de productos y servicios, que son protegidos por los mecanismos y normas que otorga la propiedad industrial, que garantizan su uso y aprovechamiento en forma exclusiva y temporal.

Sin embargo, de los beneficios descritos anteriormente, la desigual posesión de conocimiento, efectivamente, ocasiona procesos de crecimiento heterogéneos, al respecto el hindú Vandana Shiva, señala que para los países en desarrollo, las patentes representan las herramientas de una nueva 
colonización, pero los países occidentales desarrollados, las consideran un derecho "natural", como lo fue la conquista durante el colonialismo, diferenciando que entre la colonización de ayer y la de hoy, actualmente, lo que hay que controlar son los sistemas económicos y los mercados. El conocimiento en sí tiene que ser convertido en propiedad (Ramos, 2001; Iglesias, 2006). Lo que está en juego en los conflictos y controversias sobre las patentes es la conquista secular de los diversos sistemas de conocimiento y economía; el autor presenta una crítica válida a lo que denomina espías, delitos y derechos de propiedad intelectual (Valdana, 2003).

Otros autores en cambio, indican que las patentes son un arma de doble filo, por un lado plantea elementos positivos, y por otro, negativos, de cualquier forma, estas contribuyen a mejorar los incentivos para inventar, divulgar y transferir la tecnología, pero también generan costes para la sociedad en términos de rentas monopolísticas y barreras para el acceso y uso del conocimiento (Encaoua et al., 2010). Es en este sentido, que el desarrollo de la sociedad del conocimiento y sus efectos en la equidad global, plantea las necesidades de desarrollo, acceso y uso (Guix y Torrents, 1991; Robles, y Giner, 2008). La Declaración de Santo Domingo, adoptada durante la Asamblea General de la OEA en el 2006 afirma que el desarrollo y el acceso universal y equitativo a la Sociedad del Conocimiento constituye un desafío y una oportunidad que ayuda a alcanzar las metas sociales, económicas y políticas de los países de las Américas, denotando así, la importancia que tiene el conocimiento y la necesidad de hacer de él un mecanismo para el desarrollo de las organizaciones, consecuentemente, el desarrollo económico de los países.

No es menos cierto que la propiedad industrial considerado como el conjunto de derechos exclusivos que posee una persona natural o jurídica sobre su invención, que protegen tanto la actividad innovadora manifestada en nuevos productos, nuevos procedimientos o nuevos diseños, como la actividad mercantil, mediante la identificación en exclusiva de productos y servicios ofrecidos en el mercado (García, 1981; Arias, 2010; Pastor, 2013), permite estimular la creatividad de los investigadores e inventores, siendo la forma mayormente empleada para proteger y gestionar los activos intangibles de las personas naturales y jurídicas que participan en la actividad económica. Sin embargo, la utilización de la propiedad industrial debe estar enmarcada en un equilibrio difícil de encontrar entre las necesidades sociales y el ejercicio de la justicia. La aplicación de sistemas como las patentes, éste ha permitido en muchos casos, mantener e incrementar un nuevo colonialismo tecnológico, ello establece la relación entre número de solicitudes de patentes presentadas en la OMPI y la situación de desarrollo de los países, también descrito anteriormente. Así en el año 2014, las solicitudes en Asia fueron de 1.607.500, en Europa 346.200, 
en Norte América 614.00, en América latina 64.100 y finalmente en África 14.900 solicitudes.

Es importante destacar el aporte teórico y científico de varios investigadores, que ratifican que la propiedad industrial tiene una relación con el crecimiento económico, sin duda, la propiedad industrial representa una importante fuente de ingresos adicionales para las empresas o instituciones a través de la creación de licencias y comercialización de productos o servicios (Vargas, 2010).

Así también, habría que señalar, que la propiedad industrial no ha podido abstraerse al debate mundial respecto del factor ético de su existencia y plena vigencia como un elemento que fomenta la privatización y la comercialización del saber, así, Echeverry y Franco (2012), señalan que en cuanto a la propiedad intelectual existen dos corrientes de pensamiento claramente diferenciadas: aquellos que defienden los derechos de propiedad intelectual y aquellos que defienden los bienes intelectuales como un patrimonio de la humanidad. Consideramos que la propiedad intelectual es un asunto inexorable en las actuales condiciones de la economía mundial. La libertad intelectual entendida como la ausencia de derechos de propiedad es un asunto peligroso en la coyuntura actual, para los titulares del correspondiente derecho de propiedad industrial representa su invención, y justifica la necesidad de articular los instrumentos para la tutela que preserven el correcto ejercicio y que, como consecuencia, impulsen la actividad de la investigación para el desarrollo (Gómez, 2012).

Autores como López-Cozar y Cuello de Oro, (2008) afirman que tendría mucho sentido invertir importantes sumas económicas en un esfuerzo innovador si no se estableciesen mecanismos que permitan asegurar la recuperación y rentabilidad de los recursos invertidos una vez desarrollada e implantada la invención. En la misma línea, otros autores señalan que se necesita conferir derechos de protección de propiedad intelectual, especialmente patentes de invención, como mecanismo para el progreso de la actividad investigativa. Éste constituye el fundamento económico del actual sistema de patentes (Gómez, 2003).

\section{Conclusion}

El trabajo de investigación determina la importancia de los derechos de propiedad industrial desde la teoría y las experiencias de los investigadores en los centros de educación superior de Ecuador, que hacen especial énfasis entre la práctica de la propiedad intelectual con el crecimiento y desarrollo económico de los países. Establece por otra parte, que en países desarrollados la generación de la innovación y el aprovechamiento de los derechos que genera la propiedad industrial son una prioridad y un insumo importante para el crecimiento del sector empresarial 
y en consecuencia, el crecimiento económico. Por otra parte, se destaca el fomento a la inversión en investigación y desarrollo, como un aspecto diferenciador, que en la práctica genera la propiedad industrial, que sin duda, como consecuencia última, permite el desarrollo de los países.

Asimismo, la titularidad de los derechos de propiedad industrial ha permitido el desarrollo heterogéneo de los países, no por vicios o defectos que ésta contenga, sino por el uso abusivo que varios países han realizado de aquellos derechos de propiedad industrial, ante la falta de regulaciones supranacionales que lo impidan y porque se ha homogenizado sus normas de protección a nivel de todos de los países, sin tener en cuenta su diferente grado de desarrollo tecnológico, económico y cultural; lo cual se podría evitar poniendo limitaciones a los derechos de propiedad industrial en las legislaciones nacionales de los países y aprovechando eficientemente la flexibilidad que otorgan los diferentes convenios que firman las naciones a nivel mundial.

Finalmente, en el contexto de la globalización es pertinente que los centros de educación superior, como son las Universidades implementen un modelo de protección de propiedad intelectual que les permita generar conocimiento en base a la innovación y creación de prototipos, ideas, productos, bienes y servicios puesta al servicio de la sociedad.

\section{References:}

1. Altés, J. (2015). Investigación, docencia universitaria y derechos de propiedad intelectual, Edit. Tirant Lo Blanch, España.

2. Alvarez, C. (1969). Propiedad intelectual, Boletín de la ANABAD, $\mathrm{N}^{\mathrm{o}}$ 55, vol. 1, págs. 14-16.

3. Arias, V. (2010). Propiedad industrial e intelectual, Veinticinco años de impacto del Derecho comunitario en el Derecho español / Carlos Francisco Molina del Pozo (dir.), págs. 255-274

4. Belly, P. (2004). El shock del magnament, la revolución del conocimiento, McGraw-Hill / Interamericana de México, México.

5. Bueno, E. (2011). Modelo Intellectus: Medición y gestión del capital intelectual, Centro de Investigación sobre la Sociedad del Conocimiento, Madrid, España.

6. Casado, C. (1991). Interacción entre Propiedad Industrial y Propiedad Intelectual, Derechos de autor y derecho conexos en los umbrales del año 2000, SOLIAS, Vol. 1, págs. 81-94.

7. Caryannis, E. (2016), Measuring intangibles managin for tangible outcomes in research and innovation, International Journal of Nuclear Knowledge Management, The George Washington University, Washington D.C. 
8. David, P. y Foray, D. (2002). La sociedad del conocimiento, Documento internacional de Ciencias Sociales, UNESCO.

9. Encaoua, D.; Guellec, D.; Dominique, J. y Martínez, C. (2010), Sistema de patentes para fomentar la innovación: lecciones de análisis económico, Instituto de Políticas y Bienes Públicos (IPP), CCHS-CSIC, Madrid, España.

10. Franco, L. y Echeverri, R. (2012). Valoración de tecnología para efectos de negociación, Fondo Editorial ITM, Medellín, Colombia.

11. García, R. (1981). La propiedad industrial e intelectual, Revista de derecho mercantil, $\mathrm{N}^{\circ} 161-162,1981$, págs. 625-650

12. García, J. (2010). Gestión de la innovación empresarial. Claves para ser una empresa innovadora, Editorial Netbiblo, S.L. La Coruña, España.

13. Gómez, M. (2012). La tutela penal de los derechos sobre bienes inmateriales los delitos contra la propiedad intelectual e industrial, Artes Gráficas, Valencia, España.

14. Gómez, X. (2003). Patentes de invención y derecho de competencia económica, Ediciones Abya-Yala, Corporación Editora Nacional, Quito, Ecuador.

15. Guix, V. y Torrents, L. (1991). Derecho de la propiedad industrial e intelectual, Revista jurídica de Catalunya, Vol. 90, № 2, págs. 557568.

16. IDRIS, Kamil (2013). La propiedad intelectual al servicio del crecimiento económico, KSR, Ginebra, Suiza.

17. Iglesias, C. (2006). Propiedad intelectual: claves de la reforma de la Ley de propiedad intelectual, Iuris: Actualidad y práctica del derecho, $\mathrm{N}^{\circ} 109$, págs. 48-53.

18. López-Cózar, C. y Cuello de Oruro, D. (2008), Cómo proteger los resultados de la innovación en la empresa, Editorial Netbiblo, La Coruña, España.

19. Martínez de Aguirre, C. (2016). La propiedad intelectual, Curso de derecho civil. Derechos reales / coord. por Pedro Valentín de Pablo Contreras, págs. 237-260.

20. Núñez Jover, J. (2012). Conocimiento y sociedad: pensando en el desarrollo. Reflexiones sobre ciencia, tecnología y sociedad, Editorial Ciencias Médicas, La Habana, Cuba.

21. Pastor, J. (2013). Creatividad e innovación factores clave para la gestión e internalización, Publicaciones ICEX, Madrid, España.

22. Pérez Lindo, A. (1998). Mutaciones: escenarios y filosofías del cambio de mundo. Edit. Biblos, Buenos Aires, Argentina.

23. Ramos, I. (2001). Propiedad industrial y propiedad intelectual, Revista de la contratación electrónica, N 12, págs. 87-100. 
24. Rogel, C. (2004). Diseños, propiedad intelectual y propiedad industrial, REGAP: Revista galega de administración pública, $\mathrm{N}^{\mathrm{o}} 36$, págs. 39-60.

25. Robles, A. y Giner, C. (2008). Competencia. Propiedad industrial e intelectual, Derecho de los negocios, Año no 19, Nº 210, págs. 55-57.

26. UNESCO, (2005), Hacia las sociedades del conocimiento, Informe Mundial de la UNESCO, Organización de las Naciones Unidas para la Educación, la Ciencia y la Cultura, Paris, Francia.

27. Valhondo, D. (2010). Gestión del conocimiento del mito a la realidad, Ediciones Días de Santos, S. A., Madrid, España.

28. Vargas, J. (2010). La propiedad industrial y su importancia en el comercio, Dirección de Relaciones Internacionales del Instituto Mexicano de Propiedad Industrial, México D.F., México. 